\title{
BLE Broadcasting Impact in a Real Network Environment
}

\author{
Miran Borić \\ Ana Fernández Vilas \\ Rebeca P. Díaz Redondo \\ Department of Telematics Engineering Department of Telematics Engineering Department of Telematics Engineering \\ E.E. Telecomunicación Campus E.E. Telecomunicación Campus E. Telecomunicación Campus \\ universitario 36310 Vigo, Pontevedra universitario 36310 Vigo, Pontevedra universitario 36310 Vigo, Pontevedra \\ Spain \\ +34986818704 \\ Spain \\ +34986813868 \\ mboric@det.uvigo.es \\ avilas@det.uvigo.es \\ Spain \\ +34986813469 \\ rebeca@det.uvigo.es
}

\begin{abstract}
Bluetooth Low Energy is taking over small mobile device world by gaining importance in many aspects of human social life. In this paper, we are investigating the potential impact of BLE devices broadcasting availability and what are the differences according to different real environment broadcasting scenarios. By means of experiments, we conduct several scenarios depending on two variables - duration of device advertising and a number of advertising devices. We found that the duration of advertisement is not affecting message delivery when the advertiser is alone in the network. Also, we predict future growth of network latency. We found that this behaviour is happening in the case when devices are in a closer range of advertising.
\end{abstract}

\section{Categories and Subject Descriptors}

C.4. [Performance of Systems]: Measurement techniques.

\section{General Terms}

Measurement, Performance, Experimentation.

\section{Keywords}

Bluetooth Low Energy, Broadcasting, Beacons, Internet of Things, Experiments.

\section{INTRODUCTION}

Bluetooth low energy (BLE) technology is a relatively new technology and has already found its place in many aspects of computer networking world[1]. The reason for that is a mechanism highly optimized for low energy networking operations, as the name suggests[2]. As a perfect low energy data broadcaster it presents a potential technology that could become basic approach to many different mobility scenarios and message dissemination in a real time environment area. With that in mind, the doors to user connectable devices are widely open, especially with the latest BLE concept called Bluetooth proximity beacons, often referred to as the Beacons, where LE peripherals advertise

Permission to make digital or hard copies of all or part of this work for personal or classroom use is granted without fee provided that copies are not made or distributed for profit or commercial advantage and that copies bear this notice and the full citation on the first page. Copyrights for components of this work owned by others than ACM must be honored. Abstracting with credit is permitted. To copy otherwise, or republish, to post on servers or to redistribute to lists, requires prior specific permission and/or a fee. Request permissions from Permissions@acm.org.

ICC '17, March 22 2017, Cambridge, United Kingdom

(C) 2017 ACM. ISBN 978-1-4503-4774-7/17/03 ...\$15.00

DOI: http://dx.doi.org/10.1145/3018896.3018949 themselves to the nearby devices by using advertisement packets[3]. This concept of Beacons and advertising data is part of the novel paradigm called the Internet of Things[4] where physical world is connected to the digital world. In such system, services and content will be constantly available, building the way for the new applications and enabling new ways of how we play, work and live. $\operatorname{In}[5]$ predictions say that in the next 5 years markets will generate bigger volumes of BLE beacon devices, and by that, over 60 million unit market in 2019 will be present.

However, some questions arise, when does this kind of network concept and how, becomes delay prone when many devices are broadcasting in certain area. How does the broadcasting time of devices effects the delay in the network and broadcasting of the messages. Also, small amount of simulated and the lack of real time experiments in this area motivate us to dig deeply into the Bluetooth Low Energy world and research more in the area of broadcasting, and how does it behave in crowded BLE device scenarios. This paper is trying to explain and combine broadcasting behaviour of advertising BLE channels and show the impact on the network performance according to different kinds of real broadcasting environment. For the purpose of these experiments, equipment that is used and implemented is based on Raspberry Pi 2 Model B devices and the appropriate Bluetooth low energy addition devices, explained later in the paper.

The reminder of this paper is organized in such way that Section 2 presents an overview of Bluetooth low energy and possibility of advertising data by using correct techniques. Next, in Section 3, the experimental setup is described according to the network scenario and the method of the investigation, where the Section 4 discuss about the observation end the results. Section 5 presents the conclusion and the directions of future research.

\section{BLUETOOTH LOW ENERGY OVERVIEW}

In the very beginnings of Bluetooth[6] the technology was designed and mostly used for connecting smart phones and laptops, later it was proven to be useful for transferring audio streams from smart phones to variety of audio headsets[1]. However, as the time went by, most of the user equipment were demanding bigger data throughput and faster transfer speed, Bluetooth was expanding in the same manner. In the beginning, starting with Classic Bluetooth Basic Rate (BR) with maximum data rate of 1 megabit per second (Mbps) and later with data rates up to hundreds of megabits per second[1]. 


\subsection{Bluetooth Low Energy vs. Classic Bluetooth}

According to the Bluetooth specifications (4.0 and above)[7] two technologies are defined: BR/EDR, which is classic Bluetooth, and BLE (Bluetooth Low Energy). Device can work in Singlemode where communication is enabled for BLE and does not support BR/EDR only, and Dual-mode where communication is enabled for any Bluetooth device[2]. In addition, BLE is not backward compatible with older Bluetooth technology, although devices who supports BLE can implement classic Bluetooth protocol stack in dual mode[8][2]. Bluetooth low energy (BLE) presents a short-range wireless technology built for the communication that requires low cost and low power. It is mainly developed as a single-hop communication technology, but according to[9] some advancements have been done lately in the field of BLE multi-hop area. When speaking of transfer speed and throughput, Bluetooth low energy takes totally different approach and was developed in purpose to support long battery life and to save energy[10]. Also, BLE supports multiple connections, where one device (master) can be connected to unlimited number of other devices (slaves), unlike the Classic Bluetooth which supports only 7 devices to build one piconet[11] and each connection between the devices is identified by a 32-bit access address, no further limits exists on the number of connected devices in certain BLE network connected area[12]. BLE modules use the $2.4 \mathrm{GHz}$ band and it has 37 data channels and 3 advertisement channels, with a $2 \mathrm{MHz}$ spacing between channels and GFSK (Gaussian Frequency Shift Keying) modulation[2]. The reason for this modulation is to avoid the congestion during communication, since most technologies are using the same frequency band already mentioned. Device that operates in the advertising mode transmits periodically generated advertisements by using three channels $(37,38,39)[13]$. BLE protocol stack is pretty simple to describe. It shares many similarities with classic Bluetooth and it contains Controller and the Host which presents two main parts. The Controller presents Physical layer and Link layer, which share functionality like in other network technologies. The Host part is made up of upper layers, such as Logical Link Control and Adaptation Protocol (L2CAP), the Attribute Protocol (ATT), the Generic Attribute Profile (GATT), the Security Manager Protocol (SMP) and the Generic Access Profile (GAP)(see Table 1). Connection link between Controller and Host is made through, so called, Host Controller Interface (HCI). If we investigate a little deeper into certain parts of the Controller and the Host, the GAP presents devices visibility to the outside world, and determines how device can or cannot interact with the others. It also works closely with Beacon technology, since BLE Beacons take advantage of the GAP advertising mode to interact with the outer world.

\subsection{Device Interaction and the Advertising Process}

Device interaction is done by two processes, which are Advertising and Scan Response, and once the connection is established between two devices, the process of advertising will stop and all the work is transferred to the GATT, where the data transfer process starts[2]. According to[14] and[15], it is possible to import small chunks of data into advertising channels without pairing the devices, where pairing sometimes requires manual intervention, and by that, exchange some valuable data in mobile dense scenarios, like the ones presented in[8]. With this ability, importing different types of data and sending them to nearby devices could be of great value, since it perfectly suits for many kinds of scenarios, such as: Inter-vehicular mobile communication, detecting the presents of mobile devices, discovering real world-services from the distance, etc. When speaking of PDU formats in Bluetooth Low Energy, there exits two: one that presents data packets and the other one that presents advertising. Four advertising channel types are: ADV $\backslash I N D$, ADV $\backslash$ DIRECT $\backslash I N D, A D V \backslash N O N C O N N \backslash I N D$, ADV $\backslash S C A N \backslash I N D$. The advertising process defines two kinds of events: directed and undirected. Directed advertisements are used for connecting to already known devices, where undirected advertisements, by using ADV $\backslash I N D$ packet type, are detecting unknown devices, and also contains fields like AdvA (6 bytes), that presents advertisers public or random device address and AdvData (0-31), that presents a field for optional advertising data from the advertiser. After the packet has been sent, advertiser is going to the listening mode, and that is all happening on the channel from where the advertisement is sent. After that, the advertiser, for the certain amount of time, is waiting for the response from the other side. As the BLE standard suggest, the advertiser is sending ADV $\backslash$ IND at the beginning of every advertising period by changing the channels, as well as the device that operates as a scanner[13]. Explained behaviour will be used for the experimental purposed explained further in the paper.

Table 1: Bluetooth Low Energy protocol stack

\begin{tabular}{|l|l|}
\hline Generic Access Profile & \multirow{2}{*}{ Host } \\
\hline Generic Attribute Profile & \multirow{2}{*}{ Controller } \\
\hline Attribute Protocol & \\
\hline Logical Link and Adaptation Protocol & \\
\hline Link Layer & \\
\cline { 1 - 2 } Physical Layer &
\end{tabular}

\section{SCENARIOS SETUP}

This section presents and explains scenarios conducted for the purpose of this paper. Scenarios are separated and presented by two parameters: distance of the broadcasting devices and number of the devices broadcasting. Each scenario contains 10 devices that are able to broadcast Bluetooth Low Energy advertisements and one device that has a role of collecting those advertisements (BLE data sniffer). In the experiments all devices were static and no movement has been done while data is collected. One more thing to be mentioned is the fact that all scenarios were conducted in a real room-size area (see Figure 1, 2, 3 and 4). Equipment used for the scenarios is based on Raspberry Pi 2 Model B device, Bluetooth 4.0 CSR8510 USB Module and Bluefruit LE Sniffer nRF51822. Also, in order to conduct the experiment, several BLE libraries ${ }^{1}$ had to be implemented to devices, since Raspberry Pi operating system does not support specific libraries for the purpose of Bluetooth Low Energy advertising.

\subsection{One Advertiser}

First two scenarios are conducted in such way where only one advertiser is present in the network area and broadcasting the data (see Figure 1 and Figure 2). Difference between these two scenarios is the distance of the advertising device and the BLE sniffer who is collecting those advertisements, which is 10 meters

1 libdbus-1-dev, libdbus-glib-1-dev, libglib2.0-dev, libical-dev, libreadline-dev, libudev-dev, libusb-dev. 
in first case (see Figure 1) and 1 meter in second case (see Figure 2). In each scenario, the advertisements of a broadcasting device have been activated during different periods of time, where advertiser is broadcasting its data for one minute in the beginning and then stops to broadcast for 30 seconds (standby period). Each new broadcasting period is extended by 60 seconds. After the last broadcasting period of 5 minutes broadcasting stops, where nonbroadcasting period stays the same (30 seconds) (see Figure 1 and Figure

$2)$.

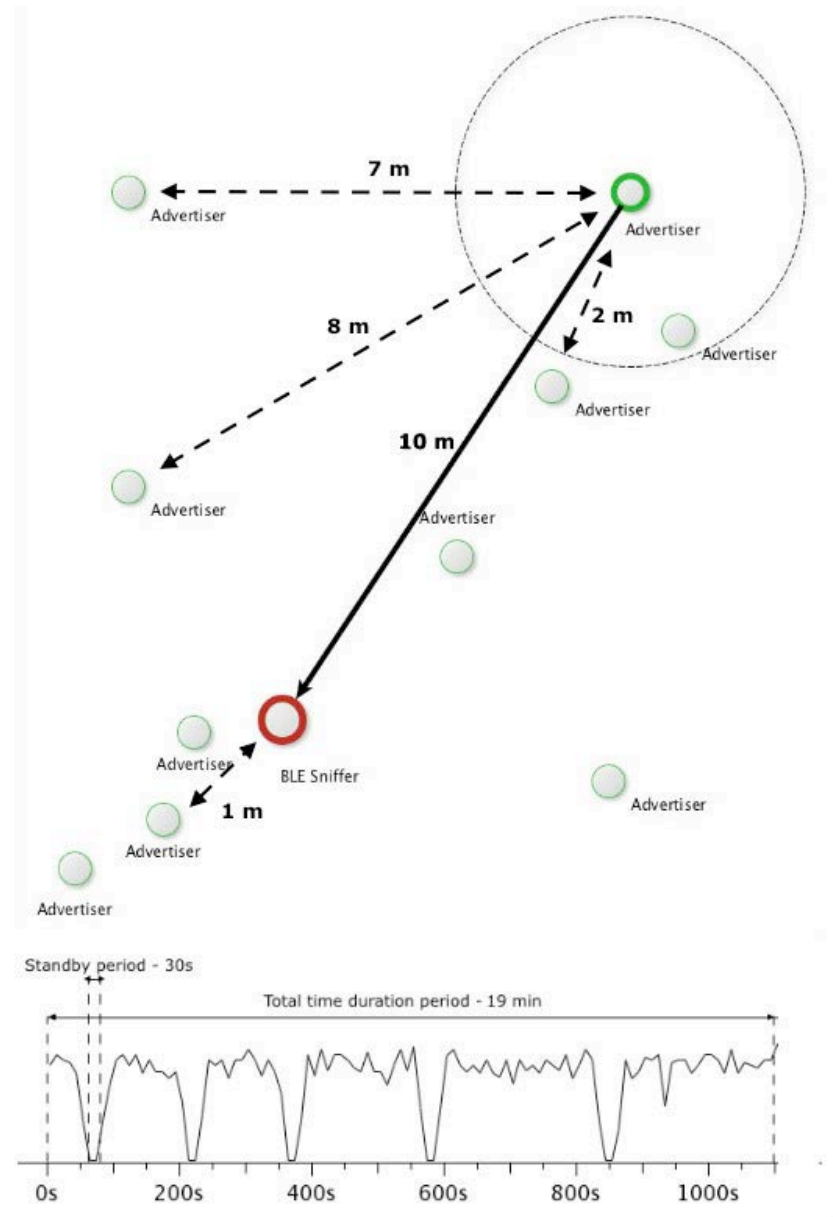

Figure 1. One advertiser - $10 \mathrm{~m}$ distance
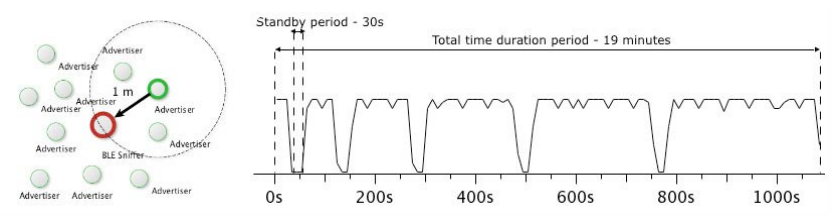

Figure2. One advertiser - $1 \mathrm{~m}$ distance

\subsection{Multiple Advertisers}

In the next scenarios, other advertisers are also included in the network and broadcasting data constantly, except one advertiser. That advertiser is acting the same like the one from the last two cases, where is following the procedure of one-minute advertising and then 30 seconds of standby state (See Figure 3 and Figure 4).
After every standby state, device is extending its broadcasting period by one minute and proceeding with that by the end of the last period, where it is broadcasting for 5 minutes. The difference between first two cases and these ones is the fact that other devices are advertising as well and, as such, making a broadcasting storm in the selected environment (see Figure 3 and Figure 4). Also, the distance between the advertiser from which data is collected is changing, where in first case is 10 meters (see Figure 3 ) and in second case 1 meter away from the BLE sniffer (see Figure 4).

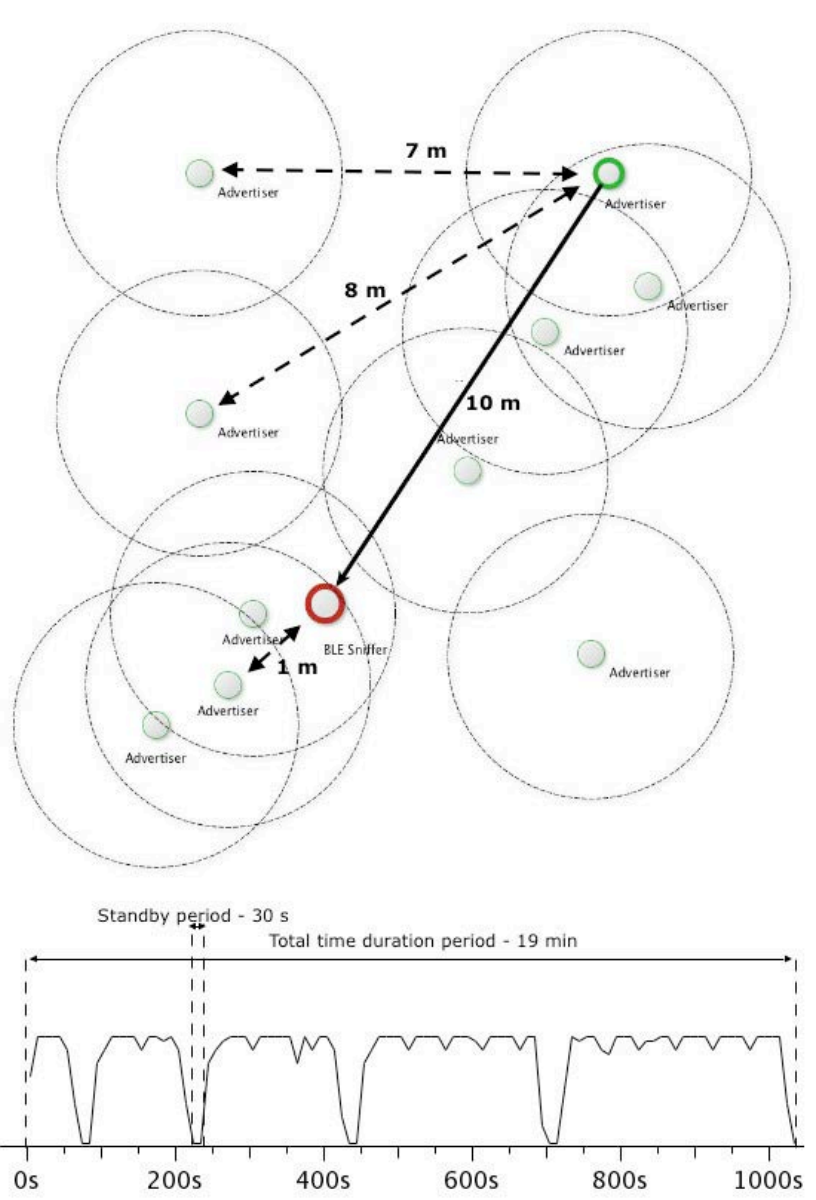

Figure 3. Multiple advertisers - $10 \mathrm{~m}$ distance
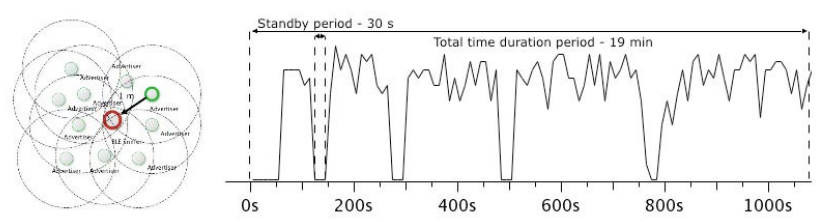

Figure 4. Multiple advertisers - $1 \mathrm{~m}$ distance

By these experiments we want to distinct the difference between several advertising scenarios where one advertiser is changing the distance from other device who is receiving and processing those advertisements. Also, we want to research the delay of packet receipt of one device while is placed in a network where other devices are advertising as well and making a potential network congestion. Each scenario is conducted during the period of approximately 19 minutes and all data has been collected directly and processed by the appropriate sniffing software[16]. Collected 
packet are based on LL LE protocol which presents one of Bluetooth protocols, closely related to scanning, connection, advertising and security from a low level.

\section{DATA OBSERVATION AND RESULTS}

\subsection{One Advertiser in the Network}

After collecting and processing all packets by the software database, the results are as follows. From the collected data of the first scenario cases, where only one advertiser is present in the network, observed device is advertising packets every 0,000410 microseconds on different channel, starting with channel 37, then 38 and finishing with 39 and that presents one advertising cycle. After that, whole cycle starts again, where delay between the cycles is around 1,2 second.

According to the first cases, when advertiser is alone in the network and broadcasting, delta time[16] of the device, is always the same (see Figure 5 and Figure 6). In our case we present network packets delay time by output of delta time, since delta time presents the time between two captured frames. Specifically, it presents the time from the beginning of the first frame to the beginning of the second frame.

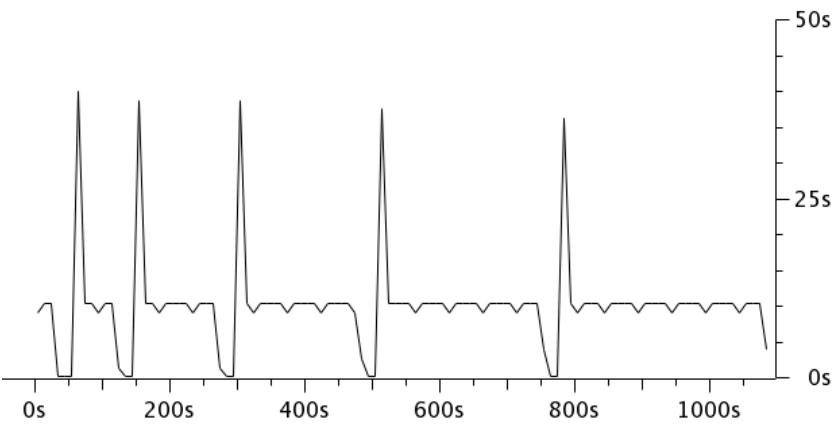

Figure 5. One advertiser scenario - $10 \mathrm{~m}$ distance - delta time

Figure 5 and Figure 6 presents a delta time sum input/output graph where $\mathrm{X}$-axis presents a tick interval of 10 seconds pick and Y-axis delta time between the collected packets. The tick interval indicates how often traffic should be plotted on the selected graph[16]. What is interesting to mention is the fact that the device, when is starting to advertise, puts its delta time to the value of around 31 seconds and then keeps it constantly around 1,2 seconds between the advertising cycles. By this, we think that the device is learning the network process by putting its delta time up to his available maximum and after 0,000410 microseconds it stabilize and proceeds with the advertisements. To the best of our knowledge no further explanation has been found regarding this behavior. From these two experiments we conclude that the duration of advertisement time, when the device is alone in the network and not affected by the others, is not affecting the delta time. Also, we conclude that the distance of devices does not affect the delta time, at least the conducted distance, when the device is advertising alone.

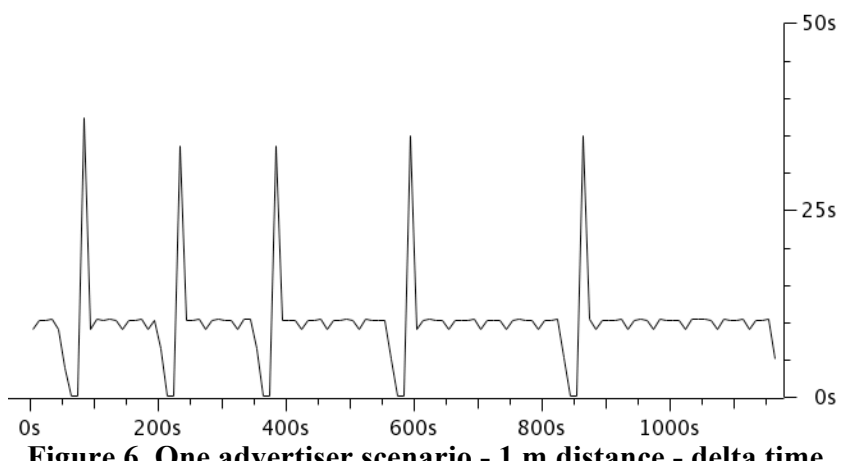

Figure 6. One advertiser scenario - $1 \mathrm{~m}$ distance - delta time

\subsection{Multiple Advertisers in the Network}

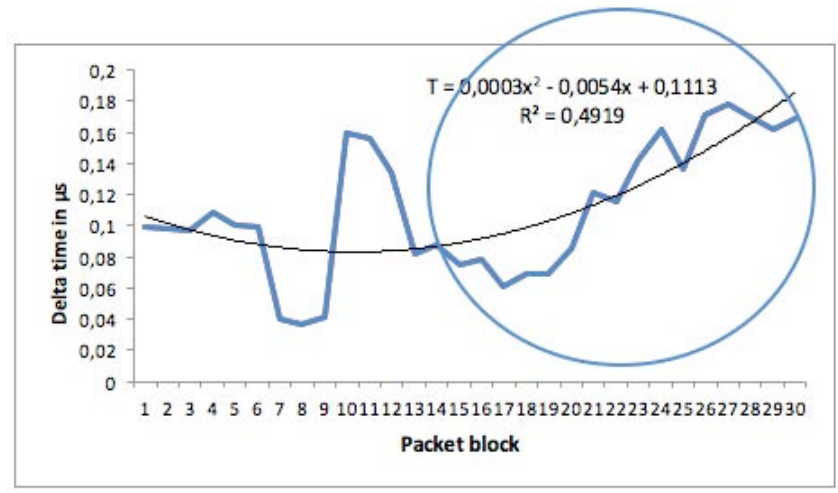

Figure 7. Multiple advertisers - $1 \mathrm{~m}$ distance - delta time during experiment

To forecast the maximum device delta time in the experiment, collected raw data from the case scenario (see Figure 4), where advertising device is not alone in the network and the distance is 1 meter between the devices, is adjusted as follows. Source data is grouped in such way that for 40 packets (approximately every 30 seconds), average delta time is calculated and separated. With this approach it is obtained totally 30 average values based on approximately 30 seconds intervals (see Figure 8). First row of the table (see Figure 8) presents number of the block packet, second row average block delta time, third raw time difference collected from the device and forth edited time difference data for easier calculating of total average time difference value. Furthermore (see Figure 7), it is clear that the first half of the diagram shows significant fluctuations, which can disturb the forecast possibility of its value. Therefore, second group of data is used for the forecast (second part of the graph) where data is considerably stable. Trend line (see Figure 9) is based exactly on these data, where the most appropriate is to select the polynomial (square) trend which shows the satisfactory quotient value of $\mathrm{R}^{2}=0.867$. In addition, in this case, we choose polynomial trend since linear trend suggest unlimited increase of delta time and by that, parameters for the following predictions are not suitable. 


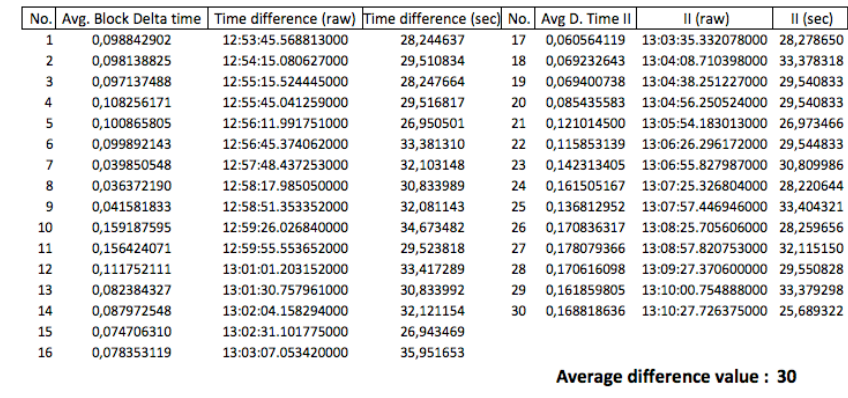

Figure 8. 30 seconds average interval block packets

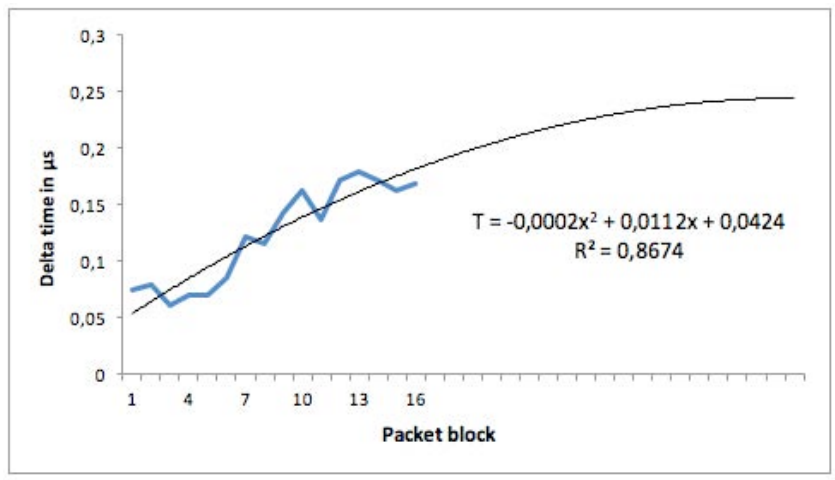

Figure 9. Multiple advertisers - $1 \mathrm{~m}$ distance second part of the graph

Based on the selected trend and by using classical differential calculation for function maximum, it can be forecast that the maximum device delta time of 0,199 microseconds would happen for $\mathrm{x}=28$, where $\mathrm{x}$ presents half-minute block interval, i.e. approximately after 840 seconds or 14 minutes of constant broadcasting (see Figure 10). It is necessary to specify that listed forecast is not reliable after a longer period of device broadcasting.

$$
\begin{aligned}
& T=-0,0002 x^{2}+0,0112 x+0,0424 \\
& T^{\prime}=-0,0004 x+0,0112 \\
& T^{\prime}=0 \text { for }-0,0004 x+0,0112=0 \\
& x=28 \\
& T(28)=0,199 \mu \mathrm{s}
\end{aligned}
$$

Figure 10. Maximum device delta time

Next case presents already explained scenario where device is not advertising alone in the network and where the distance between the device and the collector is 10 meters (see Figure 3). To forecast the maximum device delta time based on collected raw data, in this scenario packets are aggregated in 50 packet block groups by order they were coming to the device. Their order was not changed whatsoever. With this approach it is obtained totally 40 average values based on approximately 21,3 seconds intervals (see Figure 11). First row of the table (see Figure 11) presents number of the block packet, second row average block delta time, third raw time difference collected from the device and forth edited time difference data for easier calculating of total average time difference value. In the previous scenario, collected data were sorted as a 40 block packet data and here is sorted as a 50 block packet data. Since in both cases we calculate average delta time, packet block size does not affect the end result. In addition, for choosing the best trend, linear, power and exponential trend

\begin{tabular}{|c|c|c|c|c|c|c|c|}
\hline No.1 & Avg. Block Delta tir & Block time difference (ra & mentrer & & D. $\operatorname{Tim}$ & II (raw) & \begin{tabular}{|l|l|} 
II (sec) \\
\end{tabular} \\
\hline 1 & 0,00535084 & 01:07:55.5655060000 & 20,490848 & 21 & 0,042010 & 01:16:35.708322000 & 21,7744 \\
\hline 2 & 0,00579634 & 01:08:17.344614000 & 21,779108 & 22 & & & 20,4784 \\
\hline & 0,0121662 & 01:09:07.316029000 & 19,20105 & 23 & 0,035578 & 01:17:17.970250000 & 21,78353 \\
\hline & 0,02771022 & 01:09:29.065413000 & 21,749384 & 24 & 0,049389 & 01:17:39.770293000 & 21,80004 \\
\hline 5 & 0,024465 & 01:09:50.831155000 & 21,765742 & 25 & 0,050246 & 01:18:01.534977000 & 21,76468 \\
\hline 6 & 0,023530 & 01:10:11.324589000 & 20,493434 & 26 & 0,052088 & 01:18:22.036153000 & 20,50118 \\
\hline & 0,032531 & 01:10:33.091496000 & 21,766907 & 27 & 0,052208 & 01:18:43.798520000 & 21,76237 \\
\hline 8 & 0,028808 & 01:10:54.844315000 & 21,752819 & 28 & 0,058699 & 01:19:37.661284000 & 20,49083 \\
\hline & 0,011017 & 01:11:44.901532000 & 19,222756 & 29 & 0,064311 & 01:19:59.416705000 & 21,75542 \\
\hline 10 & 0,004491 & 01:12:06.671994000 & 21,770462 & 30 & 0,064117 & 01:20:21.189963000 & 21,77326 \\
\hline 11 & 0,004361 & $\begin{array}{l}01: 12: 28.458983000 \\
0\end{array}$ & 21,786989 & 31 & 0,070093 & 01:20:44.250725000 & 23,06076 \\
\hline 12 & 0,004798 & 01:12: 50.207168000 & 21,748185 & 32 & 0,067671 & 01:21:06.019438000 & 21,76871 \\
\hline 13 & 0,004313 & 01:13:10.6935244000 & 20,486356 & 33 & 0,068338 & 01:21:27.795016000 & 21,77558 \\
\hline 14 & 0,004786 & 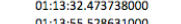 & $\begin{array}{l}21,780214 \\
2305893\end{array}$ & 34 & 0,071889 & 01:21:49.563830000 & 21,76881 \\
\hline $\begin{array}{l}15 \\
16\end{array}$ & $\begin{array}{l}0,006544 \\
0.04869\end{array}$ & 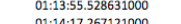 & $\begin{array}{l}23,054893 \\
273849\end{array}$ & 35 & 0,079827 & 01:22:10.072204000 & 20,50837 \\
\hline $\begin{array}{l}16 \\
17\end{array}$ & $\begin{array}{l}0,048169 \\
0,035702\end{array}$ & $\begin{array}{l}01: 14: 17.267121000 \\
0\end{array}$ & $\begin{array}{l}21,73849 \\
20,47842\end{array}$ & 36 & 0,080977 & $01: 22: 31.8325420000$ & $\begin{array}{l}21,76034 \\
2177988\end{array}$ \\
\hline & 0,035702 & $\begin{array}{l}01: 155090.89484979000 \\
011.1531 .650624000\end{array}$ & $\begin{array}{l}20,478342 \\
21,755745\end{array}$ & 38 & 0,085567 & $\begin{array}{l}01: 22: 53.6084242400 \\
01.23: 14.083705000\end{array}$ & 21,77488 \\
\hline $\begin{array}{l}18 \\
19\end{array}$ & $\begin{array}{l}0,025030 \\
0,030395\end{array}$ & $\begin{array}{l}011.15531 .650624000 \\
01.15552 .145297000\end{array}$ & $\begin{array}{l}21,755745 \\
20,494673\end{array}$ & ${ }_{39}^{38}$ & $0,0,0834891$ & $\begin{array}{l}01: 23: 144.083705000 \\
01: 23: 35.863297000\end{array}$ & $\begin{array}{l}20,477628 \\
2177959\end{array}$ \\
\hline 20 & 0,033770 & $: 16: 13.933926000$ & 1,788629 & 40 & $\begin{array}{ll}0 \\
0,091492 \\
0.09142\end{array}$ & 1:23:57.6370240000 & 21,7779373 \\
\hline & & & & & & & \\
\hline
\end{tabular}
were tested. For the best result, polynomial trend has been chosen with quotient value of $\mathrm{R}^{2}=0.9795$ (see Figure 12), since other mentioned trends were suggesting unlimited increase of the delta time.

Figure 11. 21,3 seconds average interval block packets

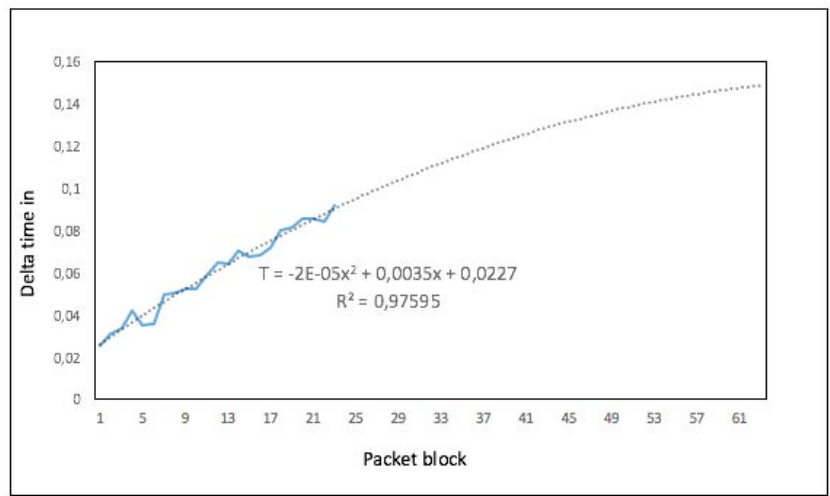

Figure 12. Fourth scenario - delta time during experiment

Following the procedure from the last case, based on the selected trend and differential calculation of function maximum (see Figure 13) we can conclude that the device is experiencing maximum delta time on block 87,5. Since the duration of one block is 21,3 seconds, maximum delta time of 0,175825 microseconds is occurring after 1863,75 second or 31 minutes of constant device advertising in a broadcasting crowded area.

$$
\begin{aligned}
& T=-0,00002 x^{2}+0,0035 x+0,0227 \\
& T^{\prime}=-0,00004+0,0035 \\
& T^{\prime}=0 \text { for }-0,00004 x+0,0035=0 \\
& x=87,5 \\
& T(87,5)=0,175825 \mu \mathrm{s}
\end{aligned}
$$

Figure 13. Maximum device delta time

When comparing last two cases, it is clear that even when devices are following the same procedure of advertising and behaving, device maximum delta time is different. The only difference in the experiments was the distance between the devices. Furthermore, bigger network latency is occurring in the scenario where devices were placed in a radius of 1 meter. In this scenario, network 
latency (maximum delta time $=0,199$ microseconds) is occurring after 14 minutes of device broadcasting, while in the scenario where the distance is greater (10 meters) network latency with maximum delta time of 0,175825 microseconds after 31 minutes of device broadcasting.

From this it can be concluded that when devices are closer to one another possibility of a faster network latency and congestion is bigger (see Figure 11 and Figure 13).

\section{CONCLUSION AND FURTHER WORK}

This paper describes different Bluetooth Low Energy scenarios conducted in a real time and environment, where the potential of advertisements impact on data delivery when one or several devices are broadcasting is explored. One part of the evaluation result show that the duration of advertisement time is not affecting the delta time, as well as the distance between the advertiser and the collector, when the device is alone in the network. On the other hand, the paper also points out two cases when the advertising of one device is affected by 10 other constant advertising devices, with different distance between the advertiser and the collector. According to this, we find that faster growth of network latency is happening in the case when devices are in a closer range of advertising. As far as we know, this work is one of the first Bluetooth Low Energy packet advertising conducted scenarios in a real time and environment, including the distance between devices, number of devices advertising and duration of advertising. This paper also opens some questions of network congestion of Bluetooth Low Energy devices in a device crowded network, since Internet of Things concept has a bright future in people's lives and it is growing rapidly. Further work includes mobility scenarios and behavior of devices while changing movement patterns, and as well as scenarios with bigger device density and amount of advertising devices. It also includes potential mobile system where through the mobile application, one or several advertisements are disseminated in a mobile network environment. Such system could present an ad-hoc mobile network, such as: city complaint system for improving people's lives in the cities, system used in disaster recovery environment, Inter-vehicular mobile environment for traffic notifications, etc.

\section{ACKNOWLEDGEMENT}

This work is funded by: the European Regional Development Fund (ERDF) and the Galician Regional Government under agreement for funding the Atlantic Research Center for Information and Communication Technologies (AtlantTIC); the Spanish Ministry of Economy and Competitiveness under the National Science Program (TEC2014-54335-C4-3-R); and the European Commission under the Erasmus Mundus Green-TechWB project (Smart and Green technologies for innovative and sustainable societies in Western Balkans; 551984-EM-1-2014-1ES-ERA MUNDUS-EMA21)

\section{REFERENCES}

[1] R. Heydon, Bluetooth low energy. Prentice Hall, 2013.

[2] K. Townsend, C. Cufí, R. Davidson, and others, Getting started with Bluetooth low energy: tools and techniques for low-power networking. O'Reilly Media, Inc., 2014.

[3] P. Martin, B.-J. Ho, N. Grupen, S. Muñoz, and M. Srivastava, "An iBeacon primer for indoor localization: demo abstract," in Proceedings of the 1st ACM Conference on Embedded Systems for Energy-Efficient Buildings, 2014, pp. 190-191.

[4] F. Xia, L. T. Yang, L. Wang, and A. Vinel, "Internet of things," Int. J. Commun. Syst., vol. 25, no. 9, p. 1101, 2012.

[5] R. ABI, "iBeacon/BLE Beacon Shipments to Break 60 Million by 2019," 2Q 2015.

[6] K. Sairam, N. Gunasekaran, and S. R. Redd, "Bluetooth in wireless communication," IEEE Commun. Mag., vol. 40, no. 6, pp. 90-96, 2002.

[7] S. I. G. Bluetooth, "Bluetooth core specification version 4.0," Specif. Bluetooth Syst., 2010.

[8] W. Bronzi, R. Frank, G. Castignani, and T. Engel, "Bluetooth low energy for inter-vehicular communications," in Vehicular Networking Conference (VNC), 2014 IEEE, 2014, pp. 215-221.

[9] K. Mikhaylov and J. Tervonen, "Multihop data transfer service for Bluetooth Low Energy," in ITS Telecommunications (ITST), 2013 13th International Conference on, 2013, pp. 319-324.

[10] S. Kamath and J. Lindh, "Measuring bluetooth low energy power consumption," Tex. Instrum. Appl. Note AN092 Dallas, 2010.

[11] J. Liu, C. Chen, Y. Ma, and Y. Xu, "Adaptive device discovery in bluetooth low energy networks," in Vehicular Technology Conference (VTC Spring), 2013 IEEE 77th, 2013, pp. 1-5.

[12] C. Gomez, J. Oller, and J. Paradells, "Overview and evaluation of bluetooth low energy: An emerging low-power wireless technology," Sensors, vol. 12, no. 9, pp. 11734$11753,2012$.

[13] K. Cho et al., "Analysis of latency performance of Bluetooth low energy (BLE) networks," Sensors, vol. 15, no. 1, pp. 5978, 2014.

[14] G. Dimitry, “'Bit-Banging' Bluetooth Low Energy - Dmitry Grinberg." [Online]. Available: http://dmitry.gr/index.php?r=05.Projects\&proj=11.\%20Bluet ooth $\% 20 \mathrm{LE} \% 20$ fakery.

[15] G. Corbellini, S. Schmid, and S. Mangold, "Two-Way Communication Protocol Using Bluetooth Low Energy Advertisement Frames," in Proceedings of the 1st International Workshop on Experiences with the Design and Implementation of Smart Objects, 2015, pp. 19-24.

[16] L. A. Chappell, Wireshark network analysis: the official Wireshark certified network analyst study guide. Protocol Analysis Institute, Chappell University, 2010. 